\title{
Computed tomography-based radiomic model predicts radiological response following stereotactic body radiation therapy in early-stage non-small-cell lung cancer and pulmonary oligo-metastases
}

\author{
Ben Man Fei Cheung, Kin Sang Lau, Victor Ho Fun Lee, To Wai Leung, Feng-Ming Spring Kong, Mai Yee Luk, \\ Kwok Keung Yuen
}

Department of Clinical Oncology, Queen Mary Hospital, Hong Kong

\author{
Received: February 27, 2021 \\ Revised: June 10, 2021 \\ Accepted: June 28, 2021 \\ Correspondence: \\ Kin Sang Lau \\ Department of Clinical Oncology, \\ Queen Mary Hospital, 1/F Professorial \\ Block, Queen Mary Hospital, Pok Fu \\ Lam Road, Hong Kong \\ Tel: +852-22554202 \\ E-mail: iamjohnnylau@gmail.com \\ ORCID: \\ https://orcid.org/0000-0002-9866-6876
}

\begin{abstract}
Purpose: Radiomic models elaborate geometric and texture features of tumors extracted from imaging to develop predictors for clinical outcomes. Stereotactic body radiation therapy (SBRT) has been increasingly applied in the ablative treatment of thoracic tumors. This study aims to identify predictors of treatment responses in patients affected by early stage non-small cell lung cancer (NSCLC) or pulmonary oligo-metastases treated with SBRT and to develop an accurate machine learning model to predict radiological response to SBRT.

Materials and Methods: Computed tomography (CT) images of 85 tumors (stage I-II NSCLC and pulmonary oligo-metastases) from 69 patients treated with SBRT were analyzed. Gross tumor volumes (GTV) were contoured on CT images. Patients that achieved complete response (CR) or partial response (PR) were defined as responders. One hundred ten radiomic features were extracted using PyRadiomics module based on the GTV. The association of features with response to SBRT was evaluated. A model using support vector machine (SVM) was then trained to predict response based solely on the extracted radiomics features. Receiver operating characteristic curves were constructed to evaluate model performance of the identified radiomic predictors.

Results: Sixty-nine patients receiving thoracic SBRT from 2008 to 2018 were retrospectively enrolled. Skewness and root mean squared were identified as radiomic predictors of response to SBRT. The SVM machine learning model developed had an accuracy of $74.8 \%$. The area under curves for $C R, P R$, and non-responder prediction were 0.86 (95\% confidence interval [Cl], 0.794-0.921), $0.946(95 \% \mathrm{Cl}$, $0.873-0.978)$, and 0.857 (95\% $\mathrm{Cl}, 0.789-0.915)$, respectively.

Conclusion: Radiomic analysis of pre-treatment CT scan is a promising tool that can predict tumor response to SBRT.
\end{abstract}

Keywords: Radiomics, Stereotactic body radiotherapy, CT, Predictor, Lung cancer, Lung metastasis

\section{Introduction}

Thoracic stereotactic body radiotherapy (SBRT) is applied for multiple indications, including early-stage and metastatic lung cancer and oligo-metastatic thoracic lesions from other tumors, allowing superi- or local control compared with conventional radiotherapy [1-3].

However, some recent experiences reported a pathological complete response (CR) rate after thoracic SBRT of about 60\%, which is lower than previously estimated [4]. Moreover, although toxicity profile of SBRT is usually fair, it is not devoid of side effects includ- 
ing pneumonitis, airway, vascular and chest wall complications $[5,6]$. The possibility to predict the responders to thoracic SBRT could thus allow a rational selection of the patients and optimize the risk/benefit ratio.

Various studies have developed models to predict clinical outcomes after thoracic SBRT for both early-stage and oligo-metastatic cancer. These studies were based mainly on clinical or conventional radiological parameters. For example, in early primary lung cancer size has been found to be a strong predictor of response [7].

Tanadini-Lang et al. [8] developed a nomogram that predicts 2-year overall survival for oligo-metastatic cancer patients that underwent SBRT. The nomogram predicts survival based on primary tumor, Karnofsky index, the control of primary, number of metastasis and tumor diameter with concordance index of 0.73. It was found that factors such as performance status, site of primary, size and number of metastases all play an important role in prognosis. For example, metastatic stage non-small cell lung cancer (NSCLC) or melanoma confers worse prognosis compared to other histologies [8]. On the other hand, smaller size, solitary metastasis and good performance status correlate with better prognosis [9].

Despite significant advancement, further improvement of predictive performance is necessary to accurately inform treatment decisions. Other than clinical features, sophisticated radiological features have been suggested to have prognostic value. With the advent of radiomics, textual features of tumors can be extracted from images such as computed tomography (CT), magnetic resonance imaging (MRI) or positron emission tomography (PET) using mathematical algorithms. The extracted features allow a comprehensive description of the radiological appearance of the lesion, identifying also characteristics that are invisible to the naked eye. Radiomic features could capture a comprehensive set of intra-tumor characteristic that may correlate with tumor aggressiveness, heterogeneity, tendency to originate metastasis and response to treatment [10].

With the increased application of SBRT for early-stage NSCLC and thoracic oligo-metastasis, enhanced predictive models based on radiomic features could facilitate to distinguish treatment responsive and non-responsive tumors. In addition, such models could be developed to be universally applicable, regardless of tumor histology or primary site.

Hence, we planned this analysis to identify predictive universal radiomic features that can predict radiological response after SBRT. We also propose a machine learning model to predict radiological response after SBRT based on radiomic features regardless of histology, primary site and staging.

\section{Materials and Methods}

\section{Patient selection}

Patients receiving thoracic SBRT using active breathing control (ABC) technique between January 2008 to December 2018 were retrospectively analyzed. The study obtained the approval of the Institutional Review Board of the University of Hong Kong/Hospital Authority Hong Kong West Cluster (HKU/HA HKW) (No. UW 16522).

Inclusion criteria were stage I NSCLC (i.e., the American Joint Committee on Cancer staged T1-2NOMO) and pulmonary oligo-metastases which had primary tumors surgically removed, had 5 or fewer lung metastases and stable systemic control.

Exclusion criteria were inability to tolerate the breath-hold required for $A B C$ technique, and thoracic tumors within the no-fly zone as defined by Timmerman et al. [11] or tumors which overlapped with mediastinal/chest wall structures. The above criteria were chosen to avoid confounding factors which make interpretation of radiomic textual features inaccurate, such as tumors with excessive movement, tumors treated with alternative SBRT dose schedules and tumors overlapping with other structures.

A total of 69 patients were recruited. All patients received 45-60 Gy in 3-5 fractions on alternate days equivalent to $B D_{10}>100$ Gy. Clinical parameters of the patients were summarized in Table 1.

Patients' breathings were actively controlled using ABC that employed a modified spirometer and occlusion valves. By closing both valves at predefined lung volume, breathing was held at a definite and stable expansion for 20 seconds while radiation beam was on. Patient was then allowed to breathe freely until the next $A B C$ cycle. CT simulation was performed using $3 \mathrm{~mm}$ slice thickness.

Treatment planning was carried out with the Eclipse radiotherapy planning system (Varian Medical Systems, Palo Alto, CA, USA). The gross tumor volume (GTV) was delineated using lung windows at width of 1,600 and level of -600. The planning target volume (PTV) was defined as GTV plus $5 \mathrm{~mm}$ margin. A 10-20 Gy per fraction was prescribed at $80 \%-90 \%$ isodose level of PTV to a total of 45-60 Gy in 3-5 fractions over 2 weeks. Prescribed dose had to cover 95\% of PTV and 99\% of PTV received > 90\% of prescribed dose. Treatment isocentre was verified before each fraction and compared to center of the tumor on $\mathrm{CT}$ image.

\section{Outcome assessment}

The primary outcome of the study was local radiological response, classified as radiological $C R$, partial response (PR), stable disease (SD), and progressive disease (PD).

Other outcomes such as locoregional failure, distant metastasis and overall survival were also studied. Survival time was calculated 
Table 1. Patient and treatment characteristics

\begin{tabular}{|c|c|c|c|c|}
\hline Characteristic & All & Non-responder & Responder & $p$-value \\
\hline Age (yr) & 73 (44-92) & $72(56-92)$ & $76(44-90)$ & 0.456 \\
\hline Sex & & & & 0.959 \\
\hline Male & 42 & 21 & 21 & \\
\hline Female & 27 & 14 & 13 & \\
\hline Initial stage & & & & 0.1315 \\
\hline Metastatic & 40 & 22 & 18 & \\
\hline Non-metastatic & 29 & 11 & 18 & \\
\hline Primary site (overall/histology) & & & & 0.099 \\
\hline Lung & 62 & 32 & 30 & \\
\hline Adenocarcinoma & - & 30 & 26 & \\
\hline Squamous cell & - & 2 & 3 & \\
\hline Small cell & - & 0 & 1 & \\
\hline Colorectal & 16 & 10 & 6 & \\
\hline Adenocarcinoma & - & 10 & 6 & \\
\hline Others & 7 & 1 & 6 & \\
\hline Hepatocellular carcinoma & - & 0 & 5 & \\
\hline Head and neck squamous cell & - & 1 & - & \\
\hline Radiation dose (Gy) & $54(45-60)$ & $54(45-54)$ & $54(50-60)$ & 0.916 \\
\hline Dose per fraction (Gy) & $18(10-20)$ & $18(12.5-18)$ & $18(10-20)$ & 0.433 \\
\hline \multicolumn{5}{|l|}{ Radiological response } \\
\hline $\mathrm{CR}$ & 31 & - & - & \\
\hline PR & 11 & - & - & \\
\hline NR & 43 & - & - & \\
\hline
\end{tabular}

Values are presented as median (range) or total number.

$C R$, complete response; PR, partial response; NR, non-responders.

from time of initial irradiation.

Regular thorax CT with contrast at 3 months interval in the first year and at 6 months interval from second year onward were performed for all patients in our tertiary institute. Additionally, PET/CT could be performed as per clinician's discretion. Tumor response was assessed by independent senior radiologists according to the Response Evaluation Criteria in Solid Tumors (RECIST) version 1.1 and Green's criteria [12]. Tumors with stable or progressive disease after SBRT were grouped together as non-responders while tumors with $C R$ and PR were grouped together as responders. Each category of response was analyzed using the best response achieved throughout the entire follow-up period.

Progression of disease within the PTV was defined as local recurrence. Locoregional recurrence was defined as recurrence in the lungs and or in regional lymph nodes. Distant metastasis was specifically defined according to the stage at diagnosis. For early-stage lung cancers, it was defined as development of lesions to distant organs or lymph nodes. For metastatic malignancies, it was defined as development of new metastases in addition to the original extent of disease before SBRT. Patients were censored regularly at least every 3 months and also when new follow-up imaging was available.

\section{Radiomic and statistical analysis}

The contoured CTs were analyzed using 3D-Slicer software. The voxel spacing of the CTs was normalized to $1 \mathrm{~mm} \times 1 \mathrm{~mm} \times 1$ $\mathrm{mm}$. Bin width of $25 \mathrm{HU}$ (Hounsfield unit) was applied to the images for radiomics feature extraction.

One hundred and ten radiomic features of the GTVs including shape, statistical and textual features were extracted using PyRadiomics module [13] (Supplementary Table S1). The extracted features were subsequently analyzed using in-house software developed in MATLAB R2020a (MathWorks Inc., Natick, MA, USA). To determine features predictive of non-responder, the receiver operating characteristic (ROC) curve was computed for individual radiomics features to distinguish non-responders (SD and PD) and responders (PR and $C R$ ). The area under curve (AUC) was calculated for each ROC curve. The significance of AUC values were evaluated using $\mathrm{z}$ test with adjustment for multiple testing by Bonferroni adjustment [14]. Cox regression were also done to elucidate the clinical factors that affects overall survival.

A model using support vector machine (SVM) was trained to predict treatment response based solely on the extracted radiomics features in accordance with the TRIPOD statement [15]. The model 
aimed to classify patients' radiological response into three classes, namely non-responder (PD and SD), PR, and CR.

In the present study, patients with NR outweighed those with PR and $C R$. As the response grouping was unbalanced, adaptive synthetic sampling method (ADASYN) was used to up-sample minority classes (PR and CR) to improve model performance. All extracted radiomics features were included in the training of the SVM model without prior selection to prevent bias. No clinical parameters were included in order to assess the ability of the model to predict response solely based on radiomic features.

Gaussian SVM was used in this study with one versus one classification technique and lasso regularization. Hyperparameters such as box constraint levels and kernel scale were optimized using Bayesian optimization during training. The hyperparameters of the best performing model would be selected. As radiomic features are highly correlated and at time redundant, lasso regularization was employed. During the process of training, weighting coefficients of different features were computed with lasso regularization. As a result, highly important features would have greater contribution towards the final model while redundant and noisy features would have little if any influence on the final model. Overfitting of SVM model can limit the generalizability of model to external datasets. Hence, internal validation through 10 -fold cross-validation was used to avoid overfitting while maintaining accuracy. ROC curves were constructed with bootstrap resampling for 1,000 times. AUC was used to evaluate model performance. Schematic representation of model training was presented in Fig. 1.

Statistical analyses were conducted by Statistical Package for the Social Sciences (SPSS) version 25.0 (IBM, Armonk, NY, USA). Statistical significance was tested using t-test for continuous variable and chi-squared test for categorical variables. Log rank test was used for univariate analysis. A p-value less than 0.05 was considered significant.

\section{Results}

\section{Clinical characteristics}

Sixty-nine patients were recruited in total from 2008 to 2018. Median age was 73 years (range, 44 to 92 years). Forty-two patients were male while 27 patients were female. Out of the 69 patients included, 55 patients had lung primary while 14 patients had oligo-metastases to the thorax from other tumors. For the 55 patients with lung primary, 29 patients had non-metastatic lung cancer, while 26 patients had metastatic lung cancer. Overall, 40 patients had metastatic disease while 29 patients had non-metastatic disease.

As some patients had more than one irradiated tumor, a total of 85 lesions were analyzed, of which 31 achieved CR, 11 achieved PR,

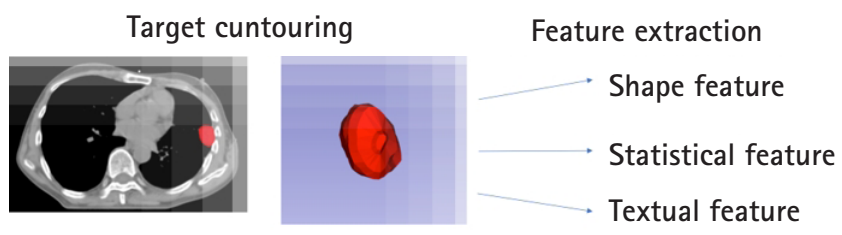

Upsampling of unbalanced outcome by ADASYN

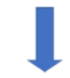

Training of Gaussian SVM with lasso regularization

Hyperparameter tuning

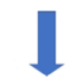

10 fold cross validation

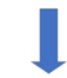

Model prediction and performance evaluation

Fig. 1. Radiomic workflow in the present study. Radiomic features (including shape, statistical, and textual features) were extracted from contoured tumor using PyRadiomics module. Under-represented population (CR and PR) was up-sampled using ADASYN algorithm. A Gaussian SVM model was then trained with lasso regularization. Hyperparameters were tuned using Bayesian optimization. Internal validation was done by 10 -fold cross-validation. The performance of the model was then evaluated and prediction generated. $C R$, complete response; $P R$, partial response; ADASYN, adaptive synthetic sampling method; SVM, support vector machine.

and 43 tumors were non-responders (NR). As 43 tumors out of 85 lesions were non-responders, the objective response rate was lower than that reported in previous studies [11]. Most extra-thoracic malignancies was colorectal in origin (16 tumors). Other extra-thoracic diseases included hepatocellular carcinoma, head and neck and esophageal cancers. In terms of histology, the large majority was represented by adenocarcinoma (72 tumors), while others were squamous cell carcinomas ( $n=7$ ), hepatocellular carcinomas $(n=5)$, and small cell carcinoma $(n=1)$.

Median follow-up time was 50.5 months (range, 1 to 132 months). Median overall survival post-SBRT was 32 months (range, 1 to 132 months; confidence interval [Cl], 24.72-39.28).

Median local failure-free survival (LFS) was 31 months (range, 1 to 132 months; $\mathrm{Cl}$, 23.6-38.4). Median locoregional failure-free survival (LRFS) was 22 months (range, 1 to 132 months; Cl, 14.46-29.54). Three-year local control rate was $87.15 \%$, while 5 -year local control rate was $80.12 \%$. Median distant metastasis-free survival (DMFS) was 18 months (range, 1 to 132 months; $\mathrm{Cl}, 10.46-25.54$ ).

For NSCLC patient, overall survival was 38 months (range, 1 to 132 months; $\mathrm{Cl}$, 29.7-46.3), LFS was 37 months (range, 1 to 132 months; $\mathrm{Cl}, 29.3-45.9$ ), LRFS was 36 months (range, 1 to 132 
months; $\mathrm{Cl}, 28.1-45.2$ ), and DMFS was 35 months (range, 1 to 132 months; $\mathrm{Cl}$, 27.1-44.2). Further subdividing patient into metastatic lung cancer and non-metastatic lung cancer. For metastatic lung cancer patients, OS was 45 months (range, 9 to 116 months; $\mathrm{Cl}$, 33-65), LFS was 36 months (range, 9 to 116 months; $\mathrm{Cl}$, 29-62), LRFS was 36 months (range, 9 to 116 months; $\mathrm{Cl}, 29-62$ ), and DMFS was 36 months (range, 9 to 116 months; $\mathrm{Cl}, 29-62$ ). For non-metastatic lung cancer patients, OS was 33 months (range, 1 to 132 months; $\mathrm{Cl}, 35-62$ ), LFS was 33 months (range, 1 to 132 months; $\mathrm{Cl}, 35-62$ ), LRFS was 33 months (1 to 132 months; $\mathrm{Cl}$, 3562), and DMFS was 33 months (range, 1 to 132 months; $\mathrm{Cl}, 35-62$ ).

Regarding non-NSCLC patients, OS was 48 months (range, 13 to 116 months; $\mathrm{Cl}, 32.6-67.3$ ), LFS was 36 months (range, 9 to 116 months; $\mathrm{Cl}, 27.8-63.7$ ), LRFS was 36 months (range, 9 to 116 months; $\mathrm{Cl}, 27.8-63.7)$, and DMFS was 30 months (range, 13 to 116 months; $\mathrm{Cl}, 14.4-45.6)$.

The median overall survival for responders and non-responders to SBRT was 47 months (range, 9 to 132 months; $\mathrm{Cl}, 36.3-57.3$ ) and 33 months (range, 1 to 93 months; $\mathrm{Cl}, 23.93-42.07$ ), respectively (Fig. 2) with trend towards improved survival for responders (CR and PR) versus non-responders to SBRT ( $p=0.059$ ).

The trend towards improved overall survival in responders versus non-responders to SBRT ( $p=0.062$ ) was confirmed by multivariate Cox regression analysis. No significant relationship with overall survival were demonstrated for other clinical characteristics (Table 2). This may be due to the small sample size in the present study. All treated tumors received SBRT dose ranging from 45-60 Gy in 3-5 fractions, equivalent to $\mathrm{BED}_{10}>100 \mathrm{~Gy}$. SBRT dose across non-responding and responding groups were similar. Patient and tumor characteristics were summarized in Table 1. All clinical characteristics including patient, disease and treatment features were not significantly different across groups.

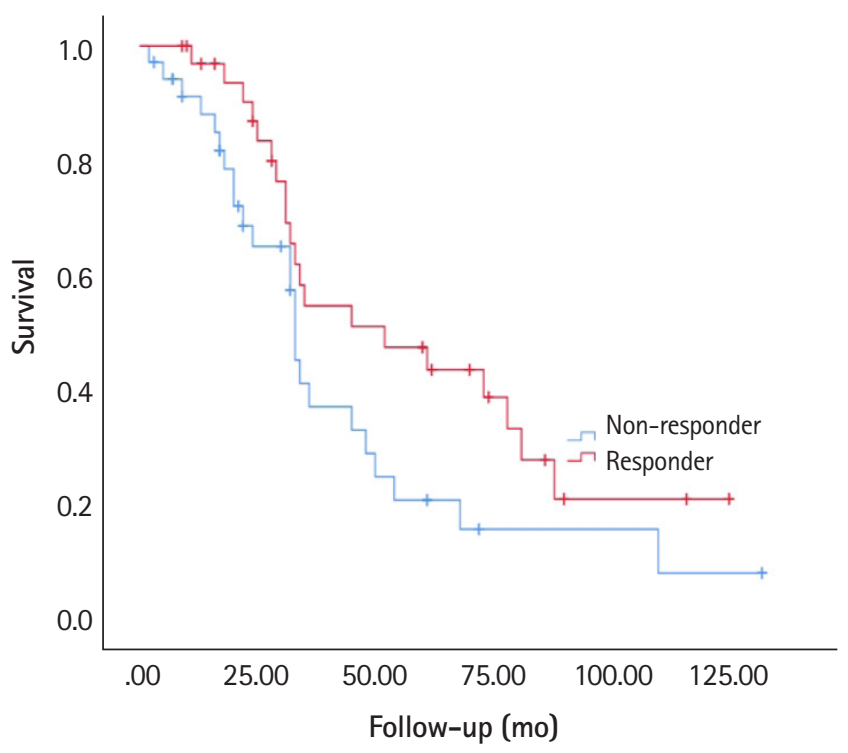

Fig. 2. Kaplan-Meier curve demonstrating trend towards improved overall survival in responders compared to non-responders to stereotactic body radiation therapy.

Table 2. Cox regression for overall survival

\begin{tabular}{|c|c|c|c|}
\hline Variable & $p$-value & $\mathrm{HR}$ & $95 \% \mathrm{Cl}$ \\
\hline Radiological response (responder vs. non-responder) & 0.062 & 2.074 & $0.965-4.458$ \\
\hline Sex (male relative to female) & 0.450 & 0.667 & $0.233-1.910$ \\
\hline Age & 0.895 & 1.002 & $0.966-1.040$ \\
\hline Smoker (smoker relative to non-smoker) & 0.422 & 0.663 & $0.243-1.809$ \\
\hline Metastasis (metastatic vs. early) & 0.262 & 0.352 & $0.057-2.180$ \\
\hline GTV volume & 0.211 & 0.972 & $0.926-1.016$ \\
\hline \multicolumn{4}{|l|}{ Histology } \\
\hline Adenocarcinoma & 0.601 & 1.608 & $0.271-9.561$ \\
\hline Squamous cell carcinoma & 0.874 & 1.239 & $0.086-17.794$ \\
\hline Hepatocellular carcinoma & 0.371 & 2.822 & $0.291-27.365$ \\
\hline \multicolumn{4}{|l|}{ Primary site } \\
\hline Lung & 0.536 & 0.412 & $0.025-6.849$ \\
\hline Colon & 0.175 & 0.133 & $0.007-2.450$ \\
\hline Liver & 0.742 & 0.584 & $0.024-14.254$ \\
\hline \multicolumn{4}{|l|}{ Radiotherapy } \\
\hline $\mathrm{BED}_{10}$ & 0.133 & 0.985 & $0.965-1.005$ \\
\hline \multicolumn{4}{|l|}{ Systemic therapy } \\
\hline Prior systemic therapy & 0.990 & 0.997 & $0.630-1.577$ \\
\hline
\end{tabular}

$\mathrm{HR}$, hazard ratio; $\mathrm{Cl}$, confidence interval; $\mathrm{GTV}$, gross tumor volume; $\mathrm{BED}_{10}$, biologically effective dose. 


\section{Radiomics features}

We first sought to determine radiomic features that can predict response to SBRT by univariate analysis. Tumors were divided into non-responders (SD and PD) and responders (CR and PR). ROC curves were constructed to predict tumor response.

Out of the 110 radiomic features, skewness and root-mean-square (RMS) were identified as statistically significant markers of NR to SBRT (AUC, 0.619-0.629; $p$ < 0.05) (Table 3). Both features are first order statistical features describing the statistical distribution of voxel intensities. Skewness is a statistical features describing the skewed distribution of voxel intensities with the region of interest. Positive skewness indicates an elongated tail to the right side of the mean on voxel intensity histogram. RMS refers to the RMS of voxel intensities within the region-of-interest (Table 3). All clinical parameters were non-predictive of treatment response ( $p>0.05)$.

\section{Multivariate machine learning model}

As the predictive accuracy of single radiomic parameter was unsatisfactory, contemporary multivariate machine learning models may be able to improve prediction accuracy. After up-sampling the minority classes (PR and CR) using ADASYN, the SVM model was trained to classify $N R, P R$, and $C R$ using one versus all technique. The overall accuracy of classification was 74.81\%. F1 scores for CR, $P R$, and NR were $0.71,0.84$, and 0.72 , respectively. The AUC for $C R$,

Table 3. Significant radiomic features in univariate analysis

\begin{tabular}{lccc}
\hline & AUC $(95 \% \mathrm{Cl})$ & $p$-value & Description \\
\hline Skewness & $0.625 \pm 0.061$ & 0.048 & $\begin{array}{c}\text { Asymmetry of voxel intensity } \\
\text { histogram }\end{array}$ \\
RMS & $0.632 \pm 0.060$ & 0.036 & RMS value of voxel intensity \\
\hline
\end{tabular}

Values are presented as mean \pm standard deviation.

$A U C$, area under curve; $\mathrm{Cl}$, confidence interval; $\mathrm{RMS}$, root-mean-square.
$\mathrm{PR}$, and NR prediction was 0.86 (95\% Cl, 0.794-0.921; $\mathrm{p}<0.05)$, 0.946 (95\% Cl, 0.873-0.978; $p<0.05)$, and 0.857 (95\% Cl, $0.789-$ 0.915; $p<0.05$ ), respectively (Fig. 3).

\section{Discussion and Conclusion}

\section{Use of radiomics to improve prognostication in SBRT}

With the advent of precision medicine, more accurate clinical models have been devised for prognostication in oncology. Most tumors are highly heterogeneous in nature in terms of its genomic expression, associated stroma and vasculature and this has profound implication on prognosis [16]. Variability in not only inter-individual, but as well remarkable among different lesions within the same individual and in different areas of the same lesion, as in metastatic tumors [17]. Hence, clinical features may not completely capture the heterogeneity of tumors.

The advent of radiomics might partially overcome this limit, as clinically undetectable features can be extracted to capture intra-tumoral and across tumor heterogeneity [10]. Hence, efforts have been made to harness radiomics for prognostication.

Huynh et al. [18] demonstrated that radiomic features can be prognostic for overall survival and distant metastasis in patients with early-stage lung cancer receiving SBRT. Multi-objective models such as random forest were constructed subsequently to improve prognostication in early stage lung cancer [19].

Apart from lung cancer, radiomic driven models have been applied in various other malignancies such as colon, prostate and breast cancer [20-22]. Both treatment outcomes and complications have been accurately predicted for various primary sites [23-25]. Specific to SBRT, clinical outcomes such as overall survival, distant metastasis or complications such as pneumonitis have been modelled using radiomics $[26,27]$. It was also applied in other imaging
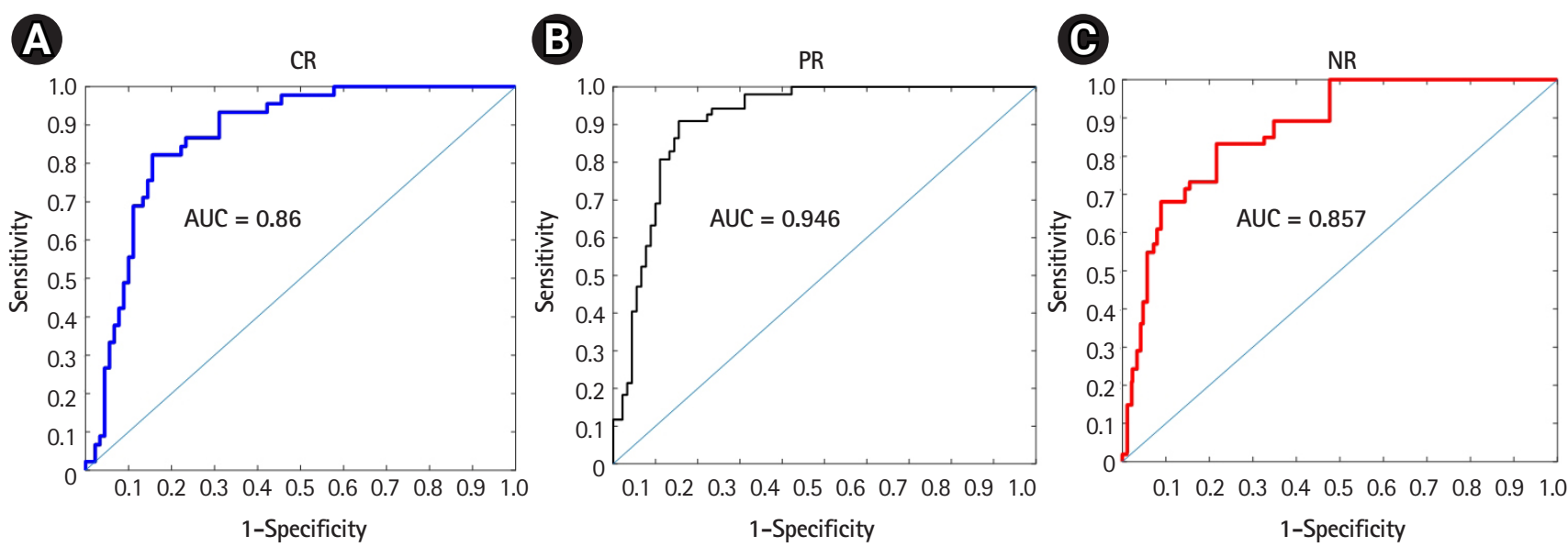

Fig. 3. Receiver operating characteristic curve for support vector machine prediction of radiological response: (A) complete response, (B) partial response, (C) non-responders. AUC, area under curve. 
modalities such as PET/CT or MRI [28].

SBRT is commonly employed for early-stage lung cancer with curative intent, and is increasingly used in patients with metastatic lung cancer with oligo-progressive or oligo-metastatic disease, allowing high local control rates [29]. This approach can achieve superior local control compared to conventional radiotherapy [30,31]. However, a pathological series by Palma et al. [4] revealed a 60\% pCR rate after SBRT for early-stage NSCLC, which is lower than previously hypothesized. The rate of local control may be lower in other unfavorable histology or in more advanced disease [32]. The choice of surgical treatment versus SBRT is at the discretion of the clinician and should be based on patient's characteristics, comorbidities and on clinical experience, preferentially in the context of a multi-disciplinary team [33].

Therefore, pre-SBRT treatment outcome prediction has pragmatic implication in the optimal selection of treatment modalities. Recent radiomic models allowed accurate prediction of overall survival, DMFS, and progression-free survival for early-stage NSCLC receiving SBRT $[18,34]$.

The population of this study included patients with both early and metastatic disease as well as different primary site and histology, with the aim is to identify universal radiomic markers of radiological response to thoracic SBRT regardless of primary site and histology.

It is of note that, despite including both metastatic and non-metastatic malignancies, there was a trend towards improved survival for radiological responders compared to non-responders. As such, radiological response may be an important prognostic marker for thoracic SBRT that could reflect an impact on overall survival. Thus, anticipating radiological response prior to administrating SBRT could play a relevant role in treatment decision.

\section{Predictive radiomics features}

To our knowledge, this is the first study recruiting patients receiving SBRT including both primary lung cancer and lung metastases from extra-thoracic disease. Skewness and RMS were identified as predictors of radiological non-responders. Interestingly, these two features were also identified in previous radiomic studies on conventional thoracic radiotherapy. Coroller et al. [35] identified seven features that were significantly associated with pathological gross residual disease following chemo-irradiation in early-stage lung cancer including skewness and RMS. These findings were confirmed by a study by Chong et al. [36] demonstrating that skewness was predictive of pathological non-responders following chemo-irradiation in lung adenocarcinoma. Considering other primary tumors, skewness was as well found to be associated with poorer prognosis in patients with colorectal cancer [37].
Changes in skewness over time were also found to be informative; longitudinal decrease in skewness post treatment was demonstrated to be predictive of better overall survival in lung adenocarcinoma [38]. As increased skewness has been found to confer poor prognosis in multiple radiomic studies across multiple tumor sites and treatment modalities, it is possible that increased skewness could be a universal negative radiomic prognostic factor. The possible underlying biological mechanisms are further discussed in the subsequent section.

Although less data exists regarding the prognostic implication of RMS, it was recently demonstrated that lower RMS was associated with poorer survival in lung cancer patients [39].

Despite enrolling a small cohort, the present study is in accordance with previous radiomic studies. In addition, the findings were confirmed in different studies utilizing different imaging and analysis protocols $[35,36,39]$. This suggests that skewness and RMS are potentially important poor radiomic prognostic factor with generalizability.

\section{Biological basis of radiomic features: radiogenomics} Increasing efforts have been made to uncover the biological basis underlying radiomic features, as biological alterations may have specific radiological appearance. Multiple radiogenomic studies have since found associations between radiomic features and gene expression, highlighting the possible link between biology and radiology [40].

Several studies indicated that skewness is associated with poorer response to radiotherapy and radio-resistance in both lung cancer and other tumor types $[34,41,42]$. Possible correlations between radiological skewness and tumor biology have been identified in previous studies. Positive skewness was found to be associated with KRAS mutations in NSCLC [43]. Indeed, lung cancers with KRAS mutations are characterized by poor prognosis and treatment resistance and same has been demonstrated in colorectal cancers as well $[44,45]$. As KRAS is a common mutation in multiple malignancies, the poorer prognosis observed in skewed tumors may be at least partially driven by KRAS mutation [46]. There are multiple mechanisms in which KRAS mutation may lead to poor prognosis, including the induction of a cancer stem cell like phenotype leading to radioresistance [47]. This may be a possible explanation for diminished response towards SBRT in patients with positive skewness in the present study.

Low FOXF2 expression was a newly discovered as an adverse prognostic factor for NSCLC [48]. FOXF2 inhibits proliferation, migration and invasion of tumor cells in vitro and was shown to enhance metastasis [49]. Perez-Morales et al. [39] demonstrated that lung tumor RMS level was correlated with FOXF2 expression. 
Hence, it is possible that radiological RMS level may reflect underlying FOXF2 expression in the tumor.

Such evidence reinforce the concept that tumor radiomic features might reflect associated underlying biological processes which ultimately affects disease prognosis and radio-sensitivity.

\section{Application of machine learning in radiomics}

Tumor textural analysis allows intralesional heterogeneity to be represented by myriad of statistical representations. Thus, datasets are typically high dimensional. In the present study, 110 radiomic features were evaluated on 85 analyzed lesions. Hypothesis testing through univariate models would necessitate multiple statistical testing. This leads to higher type I error, hence, Bonferroni adjustment would be required. Furthermore, one particular radiomic feature may not be sufficient for good classification performance. This is evident as the AUC of skewness and RMS were only 0.625 and 0.632 , respectively. The optimal decision boundary may lie in higher dimensional space which is better suited for multivariate modelling. SVM allows the decision boundary to lie within a hyperplane in high-dimensional space, as such classification performance would be significantly better [50]. SVM searches for a hyperplane that can maximally separate the outcomes of interest. Multiple studies have demonstrated the ability of SVM to classify high-dimensional clinical dataset for prediction of clinical outcomes such as development of pneumonitis or overall survival following radiotherapy [51,52]. We have demonstrated in this study that SVM was highly accurate in predicting tumor response following SBRT.

Clinical outcomes in this study were unbalanced, as patients with CR ( $n=31)$ and PR ( $n=11)$ were less than NR $(n=43)$. Despite the unbalance dataset, we were still able to identify two radiomic features that are predictive of NR which are in accordance with previous studies. In datasets with unbalanced class, the accuracy of minority class prediction is often sacrificed by the algorithm to allow higher overall accuracy [53]. Hence while building the SVM model, PR and CR were up-sampled using ADASYN to match the NR class. This approach preserves the statistical distribution of up-sampled class while increasing the statistical power [53]. Similar approach using the Synthetic Minority Oversampling Technique (SMOTE) was demonstrated in previous radiomic studies to be beneficial toward overall classification performance [19].

The application of machine learning models could have great potential in the field of radiomics and precision medicine. Machine learning allows multiple factors to be accounted in a multi-dimensional model which can be useful in the age of precision medicine. The application of radiomics and machine learning in clinical practice requires accurate and reproducible models. As radiological response to SBRT can potentially directly impact survival, the predic- tive ability of radiomic models can guide decisions to select tumors that are likely responsive to SBRT while opting for alternative therapies for more radioresistant tumors. The optimization of dedicated models could thus allow the introduction of radiomics in the clinical decision-making process.

\section{Limitations}

1) Here we present a hypothesis generating study aiming to elucidate prognostic radiomic features for future confirmation

Despite being a promising technique to provide additional prognostic information, this study is characterized by several limitations. This retrospective study enrolled a small and heterogeneous cohort which may limit its generalizability. As the study was retrospectively conducted, some factors may not be adequately controlled for. As described previously, the outcomes in each category ( $C R, P R$, and NR) were unbalanced. Although ADASYN can compensate for the imbalance statistically, genuine data points are still more preferable.

Apart from that, we acknowledge that the proportion of patients with NSCLC and non-lung tumors were unbalanced as well (55 vs. 14 patients). However, on a tumor level, there were 62 NSCLC lesions versus 23 non-lung tumors. The level of unbalance was also reduced by ADASYN to a certain extent.

Planning and treatment variability also contribute to limitation of this study. Excessive tumor movement during planning CT may limit the accuracy of feature extraction. This was addressed by recruiting only patients irradiated using $A B C$ technique to reduce tumor movement. It should also be noted that a few fractionation schedules were used within in study which may lead to differential tumor response.

This study had a strict patient selection criteria in which patients with central tumors and patients that cannot tolerate $A B C$ were excluded. This improved the accuracy of the model but at the same time limited its application in "real-world" clinical practice.

We acknowledge that different institutions utilize different imaging protocols and scanners. In addition, use of contrast or injection time may differ across institution. It has been shown that use of contrast may affect certain radiomic features [54]. Non-contrast CT thorax was employed in this study while other studies employed contrast planning CT. This may limit the generalizability and sharing of radiomic models. As such, radiomic models application may be limited to a single institution without the inclusion of multi-institutional data. A universal feature extraction protocol and feature set were also lacking, which hinders inter-study comparison. This can be overcome by data-sharing and cross-validation across different data sets which hopefully will result in more generalizable models in the future. 


\section{Conclusion}

The present study provided a proof of concept, that radiomic features can predict response to SBRT, irrespective of tumor histology, site of primary and initial staging. We also demonstrated that skewness and RMS are potential universal adverse predictive factors, in accordance with multiple previous radiomic studies. It was also shown that there was a trend towards improved overall survival tumors with a radiological response to SBRT. This validates the role of radiological response as a prognostic factor of overall survival.

The clinical implications of the definition of universal radiomic predictive features require additional prospective analysis. Further research is warranted to unravel the biological basis underlying radiomic features and treatment responsiveness.

Multivariate models such as SVM can improve classification performance allowing accurate outcome prediction. Radiomic analysis using such models can potentially be applied for treatment selection. Data sharing and unified feature extraction and processing procedures are eagerly awaited to generate more robust models in the future.

\section{Conflict of Interest}

No potential conflict of interest relevant to this article was reported.

\section{Supplementary Materials}

Supplementary materials can be found via https://doi.org/10.3857/ roj.2021.00311.

\section{References}

1. Buglione $M$, Jereczek-Fossa BA, Bonu ML, et al. Radiosurgery and fractionated stereotactic radiotherapy in oligometastatic/oligoprogressive non-small cell lung cancer patients: results of a multi-institutional series of 198 patients treated with "curative" intent. Lung Cancer 2020;141:1-8.

2. Borghetti $\mathrm{P}$, Bonu ML, Giubbolini R, et al. Concomitant radiotherapy and TKI in metastatic EGFR- or ALK-mutated non-small cell lung cancer: a multicentric analysis on behalf of AIRO lung cancer study group. Radiol Med 2019;124:662-70.

3. Ricardi U, Badellino S, Filippi AR. Stereotactic radiotherapy for early stage non-small cell lung cancer. Radiat Oncol J 2015;33: 57-65.

4. Palma DA, Nguyen TK, Louie AV, et al. Measuring the integration of stereotactic ablative radiotherapy plus surgery for early-stage non-small cell lung cancer: a phase 2 clinical trial. JAMA Oncol
2019;5:681-8.

5. Thompson M, Rosenzweig KE. The evolving toxicity profile of SBRT for lung cancer. Transl Lung Cancer Res 2019;8:48-57.

6. Kim K, Lee J, Cho Y, et al. Predictive factors of symptomatic radiation pneumonitis in primary and metastatic lung tumors treated with stereotactic ablative body radiotherapy. Radiat Oncol J 2017;35:163-71.

7. Cuaron JJ, Yorke ED, Foster A, et al. Stereotactic body radiation therapy for primary lung cancers $>3$ centimeters. J Thorac Oncol 2013;8:1396-401.

8. Tanadini-Lang S, Rieber J, Filippi AR, et al. Nomogram based overall survival prediction in stereotactic body radiotherapy for oligo-metastatic lung disease. Radiother Oncol 2017;123:182-8.

9. Fode MM, Hoyer M. Survival and prognostic factors in 321 patients treated with stereotactic body radiotherapy for oligo-metastases. Radiother Oncol 2015;114:155-60.

10. Gillies RJ, Kinahan PE, Hricak H. Radiomics: images are more than pictures, they are data. Radiology 2016;278:563-77.

11. Timmerman $R$, Paulus $R$, Galvin J, et al. Stereotactic body radiation therapy for inoperable early stage lung cancer. JAMA 2010; 303:1070-6.

12. Eisenhauer EA, Therasse $P$, Bogaerts J, et al. New response evaluation criteria in solid tumours: revised RECIST guideline (version 1.1). Eur J Cancer 2009;45:228-47.

13. van Griethuysen JJM, Fedorov A, Parmar C, et al. Computational radiomics system to decode the radiographic phenotype. Cancer Res 2017;77:e104-e7.

14. Chen SY, Feng Z, Yi X. A general introduction to adjustment for multiple comparisons. J Thorac Dis 2017;9:1725-9.

15. Collins GS, Reitsma JB, Altman DG, Moons KG. Transparent reporting of a multivariable prediction model for individual prognosis or diagnosis (TRIPOD): the TRIPOD statement. Br J Cancer 2015;112:251-9.

16. Dagogo-Jack I, Shaw AT. Tumour heterogeneity and resistance to cancer therapies. Nat Rev Clin Oncol 2018;15:81-94.

17. Nicolson GL. Generation of phenotypic diversity and progression in metastatic tumor cells. Cancer Metastasis Rev 1984;3:25-42.

18. Huynh E, Coroller TP, Narayan V, et al. CT-based radiomic analysis of stereotactic body radiation therapy patients with lung cancer. Radiother Oncol 2016;120:258-66.

19. Zhang Y, Oikonomou A, Wong A, Haider MA, Khalvati F. Radiomics-based prognosis analysis for non-small cell lung cancer. Sci Rep 2017;7:46349.

20. Tagliafico AS, Piana M, Schenone D, Lai R, Massone AM, Houssami N. Overview of radiomics in breast cancer diagnosis and prognostication. Breast 2020;49:74-80.

21. Yao $S$, Jiang $H$, Song B. Radiomics in prostate cancer: basic con- 
cepts and current state-of-the-art. Chin J Acad Radiol 2020;2: 47-55.

22. Badic B, Hatt M, Durand $S$, et al. Radiogenomics-based cancer prognosis in colorectal cancer. Sci Rep 2019;9:9743.

23. Fornacon-Wood I, Faivre-Finn C, O'Connor JP, Price GJ. Radiomics as a personalized medicine tool in lung cancer: Separating the hope from the hype. Lung Cancer 2020;146:197-208.

24. Moreira JM, Santiago I, Santinha J, et al. Challenges and promises of radiomics for rectal cancer. Curr Colorectal Cancer Rep 2019;15:175-80.

25. Gore S, Chougule T, Jagtap J, et al. A review of radiomics and deep predictive modeling in glioma characterization. Acad Radiol 2020;Jul 10 [Epub]. http://dx.doi.org/10.1016/j.acra.2020.06.016.

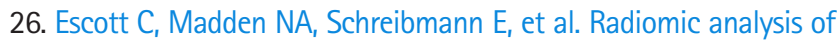
radiation induced fibrosis following stereotactic body radiation therapy. Int J Radiat Oncol Biol Phys 2019;105:E505-6.

27. Li H, Galperin-Aizenberg M, Pryma D, Simone CB 2nd, Fan Y. Unsupervised machine learning of radiomic features for predicting treatment response and overall survival of early stage non-small cell lung cancer patients treated with stereotactic body radiation therapy. Radiother Oncol 2018;129:218-26.

28. Cook GJR, Azad G, Owczarczyk K, Siddique M, Goh V. Challenges and promises of PET radiomics. Int J Radiat Oncol Biol Phys 2018;102:1083-9.

29. Nuyttens JJ, van der Voort van Zyp NC, Verhoef C, et al. Stereotactic body radiation therapy for oligometastases to the lung: a phase 2 study. Int J Radiat Oncol Biol Phys 2015;91:337-43.

30. Navarro-Martin A, Aso S, Cacicedo J, et al. Phase II trial of SBRT for stage I NSCLC: survival, local control, and lung function at 36 months. J Thorac Oncol 2016;11:1101-11.

31. Singh D, Chen $Y$, Hare MZ, et al. Local control rates with five-fraction stereotactic body radiotherapy for oligometastatic cancer to the lung. J Thorac Dis 2014;6:369-74.

32. Hamamoto $Y$, Kataoka M, Yamashita $M$, et al. Factors affecting the local control of stereotactic body radiotherapy for lung tumors including primary lung cancer and metastatic lung tumors. Jpn J Radiol 2012;30:430-4.

33. Franceschini $D$, Bruni $A$, Borghetti $P$, et al. Is multidisciplinary management possible in the treatment of lung cancer? A report from three Italian meetings. Radiol Med 2020;125:214-9.

34. Zhou Z, Folkert M, lyengar $P$, et al. Multi-objective radiomics model for predicting distant failure in lung SBRT. Phys Med Biol 2017;62:4460-78.

35. Coroller TP, Agrawal V, Narayan V, et al. Radiomic phenotype features predict pathological response in non-small cell lung cancer. Radiother Oncol 2016;119:480-6.

36. Chong Y, Kim JH, Lee HY, et al. Quantitative CT variables enabling response prediction in neoadjuvant therapy with EGFR-TKIs: are they different from those in neoadjuvant concurrent chemoradiotherapy? PLoS One 2014;9:e88598.

37. Ng F, Ganeshan B, Kozarski R, Miles KA, Goh V. Assessment of primary colorectal cancer heterogeneity by using whole-tumor texture analysis: contrast-enhanced CT texture as a biomarker of 5-year survival. Radiology 2013;266:177-84.

38. Bak SH, Park H, Sohn I, Lee SH, Ahn MJ, Lee HY. Prognostic impact of longitudinal monitoring of radiomic features in patients with advanced non-small cell lung cancer. Sci Rep 2019;9:8730.

39. Perez-Morales J, Tunali I, Stringfield O, et al. Peritumoral and intratumoral radiomic features predict survival outcomes among patients diagnosed in lung cancer screening. Sci Rep 2020;10: 10528.

40. Mazurowski MA. Radiogenomics: what it is and why it is important. J Am Coll Radiol 2015;12:862-6.

41. Yip C, Landau D, Kozarski $R$, et al. Primary esophageal cancer: heterogeneity as potential prognostic biomarker in patients treated with definitive chemotherapy and radiation therapy. Radiology 2014;270:141-8.

42. Oikonomou $A$, Khalvati $F$, Tyrrell PN, et al. Radiomics analysis at PET/CT contributes to prognosis of recurrence and survival in lung cancer treated with stereotactic body radiotherapy. Sci Rep 2018;8:4003.

43. Weiss GJ, Ganeshan B, Miles KA, et al. Noninvasive image texture analysis differentiates K-ras mutation from pan-wildtype NSCLC and is prognostic. PLoS One 2014;9:e100244.

44. Guan JL, Zhong WZ, An SJ, et al. KRAS mutation in patients with lung cancer: a predictor for poor prognosis but not for EGFR-TKIs or chemotherapy. Ann Surg Oncol 2013;20:1381-8.

45. Arrington AK, Heinrich EL, Lee $W_{1}$ et al. Prognostic and predictive roles of KRAS mutation in colorectal cancer. Int J Mol Sci 2012; 13:12153-68.

46. Prior IA, Hood FE, Hartley JL. The frequency of ras mutations in cancer. Cancer Res 2020;80:2969-74.

47. Wang M, Han J, Marcar L, et al. Radiation resistance in KRASmutated lung cancer is enabled by stem-like properties mediated by an osteopontin-EGFR pathway. Cancer Res 2017;77:2018-28.

48. Kong PZ, Li GM, Tian Y, Song B, Shi R. Decreased expression of FOXF2 as new predictor of poor prognosis in stage I non-small cell lung cancer. Oncotarget 2016;7:55601-10.

49. Wang S, Li GX, Tan CC, et al. FOXF2 reprograms breast cancer cells into bone metastasis seeds. Nat Commun 2019;10:2707.

50. Gunn SR. Support vector machines for classification and regression. Southampton, UK: School of Electronics and Computer Science, University of Southampton; 1998.

51. Chen S, Zhou S, Yin FF, Marks LB, Das SK. Investigation of the 
support vector machine algorithm to predict lung radiation-induced pneumonitis. Med Phys 2007;34:3808-14.

52. Jayasurya K, Fung G, Yu S, et al. Comparison of Bayesian network and support vector machine models for two-year survival prediction in lung cancer patients treated with radiotherapy. Med Phys 2010;37:1401-7.

53. He H, Bai Y, Garcia EA, Li S. ADASYN: adaptive synthetic sampling approach for imbalanced learning. Proceedings of 2008 IEEE In- ternational Joint Conference on Neural Networks (IEEE World Congress on Computational Intelligence); 2008 Jun 1-8; Hong Kong, China. p. 1322-8.

54. Kakino R, Nakamura M, Mitsuyoshi T, et al. Comparison of radiomic features in diagnostic CT images with and without contrast enhancement in the delayed phase for NSCLC patients. Phys Med 2020;69:176-82. 